\title{
The coffee bean sign
}

\author{
Brian Darryl Moseley • Anjali Bhagra
}

Received: 23 May 2009 / Accepted: 29 September 2009/Published online: 20 November 2009

(C) Springer-Verlag London Ltd 2009

An 89-year-old woman presented to our emergency department (ED) with a 4-day history of abdominal pain and vomiting. The physical exam revealed hypotension and a distended abdomen with generalized tenderness. Laboratory studies revealed hyperkalemia and elevated creatinine, lactate, and troponin. Given her hemodynamic instability, an emergent noncontrast abdominal computed tomography (CT) was obtained (Figs. 1 and 2).

The patient was diagnosed with sigmoid volvulus. Sigmoid volvulus is the third leading cause of large bowel obstruction, behind cancer and diverticulitis [1]. Her CT scan revealed marked distention of the sigmoid colon and rectum with abundant stool in the rectum. The coronal image demonstrated a twisted loop of sigmoid with two air-filled compartments and a central double wall ending at the point of the twist, referred to as the "coffee bean" sign. This has also been observed in abdominal X-rays and is highly suggestive of sigmoid volvulus [2]. The diagnostic accuracy of plain X-rays ranges from 30 to $90 \%$, depending on the experience of the

No grants or financial support were requested for the preparation of this manuscript.

B. D. Moseley $\cdot$ A. Bhagra

Department of Internal Medicine, Mayo Clinic,

Rochester, MN, USA

B. D. Moseley $(\square)$

200 First Street SW,

Rochester, MN 55905, USA

e-mail: moseley.brian@mayo.edu

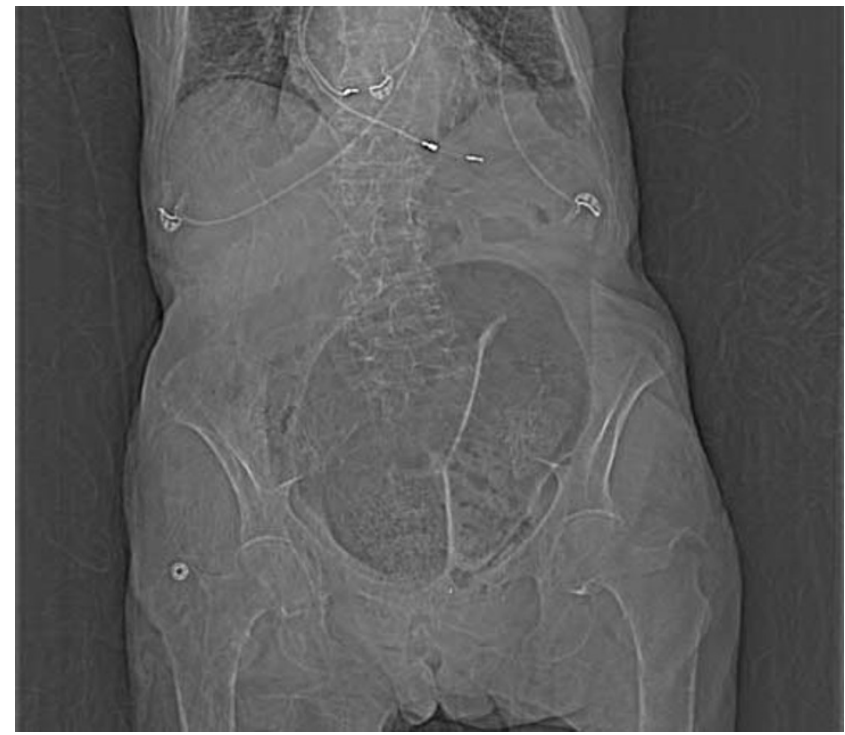

Fig. 1 Scout film of the CT abdomen, demonstrating the findings on a plain film

interpreting physician [3]. When an X-ray is equivocal, contrast enema or a CT scan can be helpful [4]. Pseudoobstruction and cecal volvulus can mimic sigmoid volvulus radiographically; however, this is rare [5].

Given the risk of gut ischemia, necrosis, and perforation, prompt management of sigmoid volvulus is warranted. Management with rectal tube placement can often be attempted. Surgery is recommended for patients with signs of bowel gangrene, peritonitis, or who fail nonoperative management [6]. Our patient and her family opted for comfort care only. 


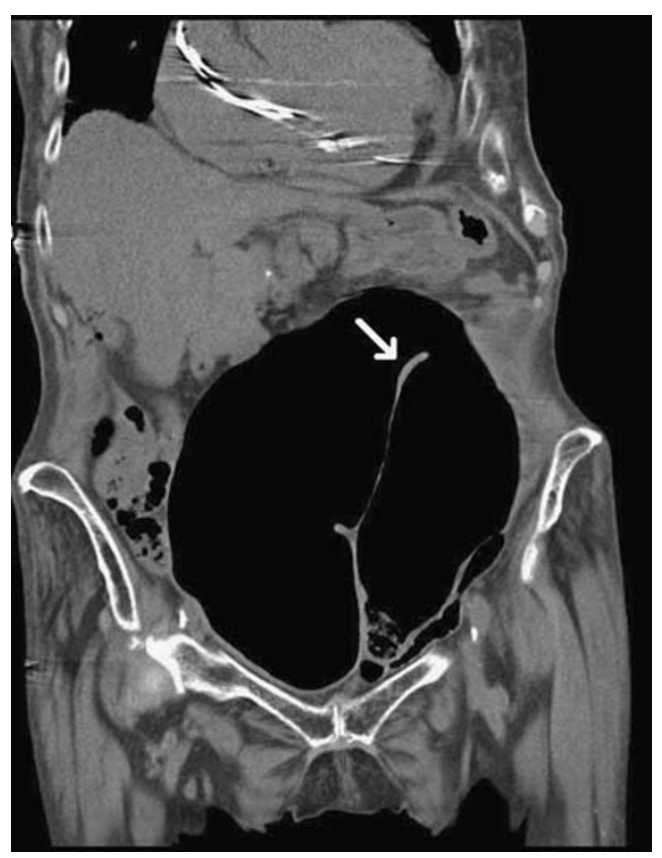

\section{References}

1. Lal SK, Morgenstern R, Vinjirayer EP, Matin A (2006) Sigmoid volvulus an update. Gastrointest Endosc Clin N Am 16(1):175-187

2. Lee YS, Lee WJ (2008) Coffee-bean sign. CMAJ 178(13):1657

3. Tiah L, Goh SH (2006) Sigmoid volvulus: diagnostic twists and turns. Eur J Emerg Med 13(2):84-87

4. Catalano O (1996) Computed tomographic appearance of sigmoid volvulus. Abdom Imaging 21(4):314-317

5. Feldman D (2000) The coffee bean sign. Radiology 216(1):178179

6. Oren D, Atamanalp SS, Aydinli B et al (2007) An algorithm for the management of sigmoid colon volvulus and the safety of primary resection: experience with 827 cases. Dis Colon Rectum 50 (4):489-497

Fig. 2 CT abdomen, revealing a twisted loop of sigmoid forming two large air-filled compartments with a central double wall ending at the point of the twist (white arrow) 